\title{
Association of dietary inflammatory potential (DIP) and endothelial function biomarkers among female nurses of Isfahan hospitals
}

\author{
Mohammad Gholizadeh ${ }^{1}$, Ebrahim Falahi², Ammar Hassanzadeh Keshteli², Ahmadreza \\ Yazdan $\mathrm{Nik}^{2}$, Parvane Saneei ${ }^{2}$, Ahmad Esmaillzadeh ${ }^{3}$, and Ahmad Saedisomeolia ${ }^{1}$ \\ ${ }^{1}$ Tehran University of Medical Sciences \\ ${ }^{2}$ Affiliation not available \\ ${ }^{3}$ Tehran University of Medical Sciences, Tehran, Iran
}

April 27, 2021

\begin{abstract}
Background Dietary inflammatory index (DIP) is a new dietary index designed to evaluate individuals' diets. In addition, adhesion molecules are important biomarkers for assessing endothelium inflammation that they related to atherosclerosis and cardiovascular disease. Also, there is no study for assessing the association between adhesion molecules and DIP until now as well as other studies that assessed the relationship between dietary inflammatory index or DIP have controversy. Aim of the Work The purpose of this cross-sectional study was to determine the correlation between DII and endothelial markers such as E-selectin, intercellular adhesion molecule-1 (sICAM-1) and vascular cell adhesion molecule-1 (sVCAM-1) among female nurses from Isfahan. In this study, dietary inflammatory potential (DIP) was used instead of DII. Patients and Methods This study was performed on 420 healthy nurses. The nurses were selected by random cluster sampling method from private and public Isfahan hospitals. A validated food frequency questionnaire (FFQ) was applied to assess the dietary inflammatory potential. A fasting blood sample was collected for measuring the plasma levels of the endothelial markers and other variables. Results After adjusting different potential confounders, no statistical association was found between DIP and sICAM-1, E-selectin and sVCAM-1 in model I ( $\mathrm{P}=0.57,0.98$ and 0.45$)$, model II $(\mathrm{P}=0.57,0.98$ and 0.45$)$ and model III $(\mathrm{P}=0.67,0.92$ and 0.50$)$ in comparison to the crude group $(\mathrm{P}=0.35,0.83$ and 0.49 , respectively). Conclusion The results revealed that the plasma levels of endothelial markers including E-selectin, sICAM-1, and sVCAM-1 were not significantly associated with DIP in female nurses.
\end{abstract}

Association of dietary inflammatory potential (DIP) and endothelial function biomarkers among female nurses of Isfahan hospitals

Abstract

\section{Background}

Dietary inflammatory index (DIP) is a new dietary index designed to evaluate individuals' diets. In addition, adhesion molecules are important biomarkers for assessing endothelium inflammation that they related to atherosclerosis and cardiovascular disease. Also, there is no study for assessing the association between adhesion molecules and DIP until now as well as other studies that assessed the relationship between dietary inflammatory index or DIP have controversy.

\section{Aim of the Work}

The purpose of this cross-sectional study was to determine the correlation between DII and endothelial markers such as E-selectin, intercellular adhesion molecule-1 (sICAM-1) and vascular cell adhesion molecule1 (sVCAM-1) among female nurses from Isfahan. In this study, dietary inflammatory potential (DIP) was used instead of DII. 


\section{Patients and Methods}

This study was performed on 420 healthy nurses. The nurses were selected by random cluster sampling method from private and public Isfahan hospitals. A validated food frequency questionnaire (FFQ) was applied to assess the dietary inflammatory potential. A fasting blood sample was collected for measuring the plasma levels of the endothelial markers and other variables.

\section{Results}

After adjusting different potential confounders, no statistical association was found between DIP and sICAM1, E-selectin and sVCAM-1 in model I ( $\mathrm{P}=0.57,0.98$ and 0.45$)$, model II ( $\mathrm{P}=0.57,0.98$ and 0.45$)$ and model III $(\mathrm{P}=0.67,0.92$ and 0.50$)$ in comparison to the crude group $(\mathrm{P}=0.35,0.83$ and 0.49 , respectively).

\section{Conclusion}

The results revealed that the plasma levels of endothelial markers including E-selectin, sICAM-1, and sVCAM-1 were not significantly associated with DIP in female nurses.

Keywords : Dietary inflammatory potential, sICAM-1, endothelial markers, sVCAM-1, E -selectin What's known?

This is a novel study in women for evaluating the association between the dietary inflammatory index and sICAM-1, sVCAM-1, and E-selectin.

One previous study that assessed the relationship between DII and adhesion molecules including ICAM-1 VCAM-1 in adolescent. They found a positive association between DII and sVCAM-1, however, similarly to our finding, they did not represent any association between DII and sICAM-1 concentrations. Moreover, some recent studies evaluated the correlation between DII and cardiovascular diseases. However, they did not find any association between DII and cardiovascular ischemic diseases, myocardial infarction in women inversely men. Although, some studies found a positive relationship between DII and carotid plaque severity, diabetic mellitus, high blood pressure, cholesterol and obesity.

\section{What's new?}

Furthermore, current study confirmed the result of Shivappa et al about ICAM-1. But, opposite to their findings, we did not observe any correlation between DII and sVCAM-1. Whereas, these two studies did not perform on the same age and sex groups.

There is no study assessing the association between DII and E-selectin.

\section{Introduction}

Atherosclerosis is a continuing inflammatory state of the vessels [1]. The progression of atherosclerosis leads to myocardial infarction and sudden death [2]. It is believed that atherosclerosis is an inflammatory condition that is largely responsible for cardiovascular disease (CVD) mortality [3, 4]. Endothelial dysfunction contributes to the pathogenesis of vascular disease and plays an important role in CVD as well $[5,6]$. Endothelial dysfunction is characterized by impaired activity of endothelial derived relaxant factors and increased activity of vasoconstrictor factors. However, cell adhesion molecules (CAM) including E-selectin, intercellular adhesion molecule-1 (sICAM-1) and vascular cell adhesion molecule-1 (sVCAM-1) accelerate atherosclerosis $[4,7-9]$.

Adhesion molecules are normally expressed by the endothelium. They also play a role in leukocyte rolling, firm adhesion, and transmigration. Furthermore, they are associated with a variety of pathophysiological processes and inflammatory disorders. Atherosclerotic lesions and fatty streaks increase the expression of sICAM-1, sVCAM-1, and P- and E-selectin on the human endothelial cells [10, 11]. E-selectin plays an important role in acute inflammation [12-14]. Moreover, sICAM-1 and sVCAM-1 are involved in chronic inflammation $[15,16]$. Leukocyte adhesion is an important component of some vascular diseases and atherogenesis. Leukocyte recruitment occurs in a multistep process and selectin, which is expressed on the activated 
endothelial cells, is involved in the initial rolling process of leukocytes $[12,17]$. The leukocyte surface has sites for selectin ligand [18]. $\beta 1$ and $\beta 2$ integrin are expressed on leukocytes and act as binding sites for sVCAM-1 or sICAM-1. Furthermore, selectin plays a role in the initial rolling process of leukocytes whereas sICAM-1 and sVCAM-1 mediate leukocyte arresting and firm adhesion [12, 18-20].

Dietary inflammatory potential (DIP) is a new dietary index designed to evaluate the individual's diets. DIP is a tool to assess the potential inflammatory and anti-inflammatory properties of a diet based on food elements. In this index, values of $+1,0$, and -1 indicate pro-inflammation, indifferent and anti-inflammation reactions, respectively [21]. Actually, DIP is a resource to assess pro-inflammatory effects of food ingredients based on anti-inflammatory functions [21,22]. DIP has been linked to a variety of systemic biomarkers such as interleukin 6 (IL-6), tumor necrosis factor alpha (TNF- $\alpha$ ), C-reactive protein (CRP) and several metabolic diseases such as CVD, cancers, and diabetes. Many studies have found that DIP is associated with the risk of metabolic syndrome and cardiovascular diseases [23-28].

Many studies have reported a positive association between DIP and CVDs [29, 30]. Due to the increase in the global risk of CVDs and related diseases in the world, it is important to find healthy dietary patterns with low inflammatory scores to tackle inflammation and CVDs. The purpose of this study was to determine the association between DIP and endothelial markers such as sICAM, sVCAM, and E-selectin in female nurses working in Isfahan hospitals.

\section{Materials and methods}

\subsection{Participants}

Four hundred and eighty healthy female nurses aged $>30$ years participated in this cross-sectional study. The participants were selected randomly from seven public and private hospitals in Isfahan, Iran. The female nurses with a history of diabetes, malignancy, infections, and CVDs were excluded. Furthermore, the subjects who did not complete the FFQ questionnaire were also excluded from the study. Finally, 420 nurses were enrolled in the study. The participants fill in a consent form based on ... university of medical sciences ethics rules for participating on this study.

The study protocol was approved by .... university of medical sciences (IR.TUMS.VCR.REC.1399.584).

\subsection{Blood sampling}

Blood samples were collected from the participants after 12 hours of fasting to measure the levels of endothelial markers, lipid profile, and fasting blood glucose. Then, the sample were centrifuged for 30-45 minute and frozen at $70^{\circ} \mathrm{C}$. The levels of sVCAM- 1 , sICAM-1, and E-selectin were measured using commercial ELISA kits (Biosource International and Bender MED Systems) according to the manufacturer's instructions. ELISA kits were also used to measure low-density lipoprotein (LDL) and high-density lipoprotein (HDL).

\subsection{Dietary inflammatory score}

The method developed by Shivappa et al was applied to calculate the DIP scores of the diets. The Food Frequency Questionnaire (FFQ) was used to determine the dietary intake [21]. In the Iranian dietary pattern, 29 out of 45 items of DII are very common, including Macronutrients (energy, carbohydrates, fat, protein, fiber), Fat (cholesterol, saturated fatty acids (SFA), monounsaturated fatty acids (MUFA), polyunsaturated fatty acids (PUFA)), Water-soluble vitamins (pyridoxine, folic acid, niacin, thiamin, ascorbic acid and riboflavin). Fat-soluble vitamins (A,D and E), Minerals (iron, magnesium, zinc, and selenium), as well as caffeine, $\beta$-carotene, onion, garlic, pepper, and black tea [31]. Other DIP items that were uncommon in the Iranian dietary regimen were omitted form the list of FFQ.

The intake of the above dietary items was adjusted according to the daily energy intake [32]. A z-score was generated for all of the 29 items of the FFQ list for each participant. For each subject, the "standard global mean" was subtracted from the mean consumed food and divided by "global standard deviation". The global means and standard deviations were obtained by the method developed by Shivappa et al [21]. To decrease the skewness of the variables, the variables were converted to a centered percentile score. This 
score was then extended by the impact for every item [21]. The DIP scores of all foods were summed to calculate the overall score. More positive values indicated a higher inflammatory dietary potential.

\subsection{Assessment of other variables}

A computerized scale was used for weight measurement (to the nearest $0.1 \mathrm{~kg}$ ). The subjects were asked to wear light clothing with no shoes. The height was also measured on the same visit day. Finally, weight $(\mathrm{kg})$ and height $(\mathrm{m})$ were used to calculate the body mass index (BMI) according to the following formula: weight $(\mathrm{kg}) /$ height $(\mathrm{m})^{2}$.

The International Physical Questionnaire was used to evaluate daily physical activity [33, 34] as MET-hour per week. The factors such as education level, family size, and economic status were inquired from all the participants to determine their socioeconomic status. Moreover, covariate data including age, marital status, menopause situation, past medical history, smoking or medication/supplementation history were selfreported by all the participants.

\subsection{Statistical analysis}

The final analysis was performed on 420 individuals. Energy adjustment of the variables was carried out using the residual method. After completing the FFQ, the data were entered into an Excel datasheet and daily dietary intakes were compared using the IBM SPSS version 26 (IBM SPSS Statistics for Win, Armonk, NY) and Nutritionist IV (N4) software. Since there were three DIP groups (tertiles), one-way ANOVA was used for continuous variables including age, body mass index (BMI), weight, waist circumference, physical activity, and systolic and diastolic blood pressure, and Pearson's chi-square test was applied to categorical variables such as oral contraceptive (OCP) use, current corticosteroid use, menopause, marital status, overweight/obesity and socioeconomic status. Similarities between the energy intakes of the participants were adjusted by linear regression. Finally, the associations between DIP and E-selectin, ICAM, and VCAM in three tertiles was analyzed using ANCOVA.

\section{Results}

The mean \pm SD age of the participants was $34.44 \pm 7.27,34.59 \pm 6.8$ and $36.47 \pm 7.4$ years in the $1^{\text {st }}, 2^{\text {nd }}$, and $3^{\text {rd }}$ tertile, respectively. The demographic characteristics of the subjects are presented in Table 1.

The distribution of the DIP score between tertiles is shown in Table 2. Large differences in DIP scores were observed for fat (P-value:0.04), riboflavin ( $\mathrm{P}$-value $<0.001)$, folic acid ( $\mathrm{P}$-value $<0.001)$, cobalamin $(\mathrm{P}-$ value $=0.005)$, ascorbic acid $(\mathrm{P}$-value $<0.001)$, vitamin $\mathrm{A}(\mathrm{P}$-value $<0.001)$, beta carotene $(\mathrm{P}$-value $<0.001)$, zinc $(\mathrm{P}$-value $<0.001)$, tea $(\mathrm{P}$-value $=0.002)$, magnesium $(\mathrm{P}$-value $<0.001)$, onion $(\mathrm{P}$-value $<0.001)$, fiber $(\mathrm{P}$ value $<0.001)$, caffeine $(\mathrm{P}$-value $=0.03), \mathrm{SAFA}(\mathrm{P}$-value $=0.02)$, and cholesterol $(\mathrm{P}$-value $<0.01)$ between the tertiles.

The mean \pm SD plasma levels of endothelial markers in different tertiles are shown in Table 3 . There was no significant association between DIP and E-selectin in the crude model $(\mathrm{P}$-value $=0.35)$ compared to model I $(\mathrm{P}$-value $=0.57)$, model II $(\mathrm{P}$-value $=0.57)$ and model III $(\mathrm{P}$-value $=0.67)$ after adjusting for potential confounders (Figure 1).

The results showed no significant association between DIP and the plasma level of sICAM-1 in the crude model (P-value: 0.83) compared to model I (P-value: 0.98), model II (P-value: 0.98) and model III (P-value: 0.92) after adjusting for potential confounders (Figure 2).

In addition, no significant association was found between DIP and the plasma level of sVCAM-1 in the crude model compared to model I (P-value: 0.49), model II (P-value: 0.45 ) and model III (P-value: 0.50) after adjusting for potential confounders (Figure 3 ).

\section{Discussion}

No association was observed between dietary inflammatory potential (DIP) and endothelial biomarkers including E-selectin, sVCAM-1 and sICAM-1 in the participants. This dissociation remained significant 
after adjusting for possible confounders. This is the first study of the association between adhesion molecules and DIP. Other studies assessed the correlation between DIP and cardiovascular disease.

Adhesion of circulating molecules, including E-selectin, sICAM-1 and sVCAM-1, plays an essential role in endothelial dysfunction and atherosclerosis [14, 35-39]. Furthermore, reactive oxygen species (ROS) activate endothelial markers by inducing E-selectin, sICAM-1 and sVCAM-1. It has been reported that sICAM1 plays an important role as a predictor of CVD [40]. Moreover, the sVCAM-1 expression represents the inflammatory conditions of the vascular walls and predicts fatal coronary artery disease in the future [39, 41]. Plasma levels of endothelial markers such as sE-selectin and sICAM-1 correlate with prognosis [11]. Many studies have assessed the correlation of DIP with CVD.

The results of the present study are consistent with a study by Imran khan et al who carried out a cohort study on 1111 subjects to evaluate the relationship between DIP and cardiovascular disease (CVD). The results showed no a significant correlation between DIP and CVD in females while a significant relationship was found in male subjects [42]. Similarly, Gabriela Pocovi-Gerardino et al conducted a cross-sectional study on 105 women with a mean age of 45.4 years old and found no significant correlation between the DIP score and CVD markers [43]. A study of 585 women aged 50-55 years old by Linda E. T. Vissers et al failed to show any correlation between DIP and CVD, ischemic heart disease, and myocardial infarction (MI) [44]. Furthermore, a prospective case-control study of 100000 participants showed no significant relationship between DII and MI [45].

By contrast, Bondonno et al reported that a high DIP score was associated with atherosclerotic vascular disease in women aged over 70 although they did not find any association between DIP and carotid plaque severity [46]. Moreover, Stefanos Tyrovolas et alcarried out a dose-dependent study to assess the correlation between DIP and CVD risk factors. They found a significant correlation between DIP and CVD risk factors such as diabetes mellitus, obesity, hypertension, and hypercholesterolemia. In addition, the participants with a high DIP score in the $3^{\text {rd }}$ and $4^{\text {th }}$ quartile had at least one CVD risk factor in comparison to the participants in the $1^{\text {st }}$ quartile [47].

It was difficult to sort out consistent results with our findings because many studies were carried out on subjects with unhealthy conditions. Moreover, there were differences between the studies in terms of the sample size. The geographic dietary pattern may also affect the results. Furthermore, many studies did not measure the plasma levels of sICAM-1, sVCAM-1 and E-selectin directly. Therefore, more studies are required to assess the correlation between endothelial markers and the DIP score.

This study had some limitations. For example, it had a cross-sectional design and therefore no conclusions can be made regarding causality. Moreover, there were some unknown confounders including shift time, bias in reporting food items, and difference in the dietary pattern between nurses in private and public hospitals, which could affect the results. Studies with larger sample sizes are required to obtain concrete results.

\section{Conclusion}

In summary, the findings suggest that the plasma levels of endothelial markers including E-selectin, ICAM-1 and sVCAM-1 have no significant correlation with dietary inflammatory potential in females.

\section{Funding sources:}

The ethics code caught by .... university of medical sciences and funding paid by .... and .... medical sciences universities.

\section{Conflict of interest:}

The authors have no conflict of interest to declare.

\section{References}

1. Hansson, G.K., A.-K.L. Robertson, and C. Söderberg-Nauclér,Inflammation and atherosclerosis. Annu. Rev. Pathol. Mech. Dis., 2006. 1 : p. 297-329. 
2. Hansson, G.K., Inflammation, atherosclerosis, and coronary artery disease. New England Journal of Medicine, 2005.352 (16): p. 1685-1695.

3. Tuttolomondo, A., et al., Atherosclerosis as an inflammatory disease. Current pharmaceutical design, 2012. 18 (28): p. 4266-4288.

4. Libby, P., P.M. Ridker, and A. Maseri, Inflammation and atherosclerosis. Circulation, 2002.105 (9): p. 1135-1143.

5. Endemann, D.H. and E.L. Schiffrin, Endothelial dysfunction.Journal of the American Society of Nephrology, 2004. 15 (8): p. 1983-1992.

6. Constans, J. and C. Conri, Circulating markers of endothelial function in cardiovascular disease. Clinica chimica acta, 2006.368 (1-2): p. 33-47.

7. Ajjan, R.A. and P.J. Grant, Cardiovascular disease prevention in patients with type 2 diabetes: The role of oral anti-diabetic agents. Diabetes and Vascular Disease Research, 2006. 3 (3): p. 147-158.

8. Kim, J.-a., et al., Reciprocal relationships between insulin resistance and endothelial dysfunction: molecular and pathophysiological mechanisms. Circulation, 2006. 113 (15): p. 1888-1904.

9. Davignon, J. and P. Ganz, Role of endothelial dysfunction in atherosclerosis. Circulation, 2004.109 (23_suppl_1): p. III-27-III-32.

10. Johnson-Tidey, R.R., et al., Increase in the adhesion molecule P-selectin in endothelium overlying atherosclerotic plaques. Coexpression with intercellular adhesion molecule-1. The American journal of pathology, 1994. 144 (5): p. 952.

11. Tenaglia, A.N., et al., Levels of expression of P-selectin, E-selectin, and intercellular adhesion molecule-1 in coronary atherectomy specimens from patients with stable and unstable angina pectoris. The American journal of cardiology, 1997. 79 (6): p. 742-747.

12. Vestweber, D. and J.E. Blanks, Mechanisms that regulate the function of the selectins and their ligands. Physiological reviews, 1999. 79 (1): p. 181-213.

13. Ley, K., Molecular mechanisms of leukocyte recruitment in the inflammatory process. Cardiovascular research, 1996. 32 (4): p. 733-742.

14. Kozuka, K., et al., Endothelial markers and adhesion molecules in acute ischemic stroke-sequential change and differences in stroke subtype. Atherosclerosis, 2002.161 (1): p. 161-168.

15. Bouhlel, M., B. Staels, and G. Chinetti-Gbaguidi, Peroxisome proliferator-activated receptors-from active regulators of macrophage biology to pharmacological targets in the treatment of cardiovascular disease. Journal of internal medicine, 2008. 263 (1): p. 28-42.

16. Abe, Y., K. Sugisaki, and A.M. Dannenberg Jr, Rabbit vascular endothelial adhesion molecules: ELAM-1 is most elevated in acute inflammation, whereas VCAM-1 and ICAM-1 predominate in chronic inflammation. Journal of leukocyte biology, 1996. 60 (6): p. 692-703.

17. Zhang, J., et al., Regulation of endothelial cell adhesion molecule expression by mast cells, macrophages, and neutrophils. PloS one, 2011. 6 (1): p. e14525.

18. Varki, A., Selectin ligands. Proceedings of the National Academy of Sciences, 1994.91 (16): p. $7390-7397$.

19. Collins, R.G., et al., P-selectin or intercellular adhesion molecule (ICAM)-1 deficiency substantially protects against atherosclerosis in apolipoprotein E-deficient mice. The Journal of experimental medicine, 2000. 191 (1): p. 189-194. 
20. Takahashi, K., M. Takeya, and N. Sakashita, Multifunctional roles of macrophages in the development and progression of atherosclerosis in humans and experimental animals. Medical electron microscopy, 2002. 35 (4): p. 179-203.

21. Shivappa, N., et al., Designing and developing a literature-derived, population-based dietary inflammatory index. Public health nutrition, 2014. 17 (8): p. 1689-1696.

22. Cavicchia, P.P., et al., A new dietary inflammatory index predicts interval changes in serum highsensitivity C-reactive protein.The Journal of nutrition, 2009. 139 (12): p. 2365-2372.

23. Shivappa, N., et al., A population-based dietary inflammatory index predicts levels of C-reactive protein in the Seasonal Variation of Blood Cholesterol Study (SEASONS). Public health nutrition, 2014.17 (8): p. 1825-1833.

24. Wirth, M., et al., Association of a dietary inflammatory index with inflammatory indices and the metabolic syndrome among police officers. Journal of occupational and environmental medicine/American College of Occupational and Environmental Medicine, 2014.56 (9): p. 986.

25. Wood, L.G., et al., Dietary inflammatory index is related to asthma risk, lung function and systemic inflammation in asthma.Clinical \& Experimental Allergy, 2015. 45 (1): p. 177-183.

26. Garcia-Calzon, S., et al., Dietary inflammatory index and telomere length in subjects with a high cardiovascular disease risk from the PREDIMED-NAVARRA study: cross-sectional and longitudinal analyses over 5 y. The American journal of clinical nutrition, 2015.102 (4): p. 897-904.

27. Neufcourt, L., et al., Prospective association between the dietary inflammatory index and metabolic syndrome: Findings from the SU. VI. MAX study. Nutrition, Metabolism and Cardiovascular Diseases, 2015.25 (11): p. 988-996.

28. Tabung, F.K., et al., Construct validation of the dietary inflammatory index among postmenopausal women. Annals of epidemiology, 2015. 25 (6): p. 398-405.

29. Namazi, N., B. Larijani, and L. Azadbakht, Dietary inflammatory index and its association with the risk of cardiovascular diseases, metabolic syndrome, and mortality: A systematic review and meta-analysis. Hormone and Metabolic Research, 2018. 50 (05): p. 345-358.

30. Ruiz-Canela, M., M. Bes-Rastrollo, and M.A. Martinez-Gonzalez, The role of dietary inflammatory index in cardiovascular disease, metabolic syndrome and mortality. International journal of molecular sciences, 2016. 17 (8): p. 1265.

31. Asadi, Z., et al., Association between dietary inflammatory index and risk of cardiovascular disease in the Mashhad stroke and heart atherosclerotic disorder study population. IUBMB life, 2020.72 (4): p. 706-715.

32. Willett, W. and M.J. Stampfer, Total energy intake: implications for epidemiologic analyses. American journal of epidemiology, 1986. 124 (1): p. 17-27.

33. Booth, M., Assessment of physical activity: an international perspective. Research quarterly for exercise and sport, 2000.71 (sup2): p. 114-120.

34. Committee, I.R., Guidelines for data processing and analysis of the International Physical Activity Questionnaire (IPAQ)-short and long forms. http://www. ipaq. ki. se/scoring. pdf, 2005.

35. Hwang, S.-J., et al., Circulating adhesion molecules VCAM-1, ICAM-1, and E-selectin in carotid atherosclerosis and incident coronary heart disease cases: the Atherosclerosis Risk In Communities (ARIC) study. Circulation, 1997. 96 (12): p. 4219-4225.

36. Ridker, P.M., et al., Plasma concentration of soluble intercellular adhesion molecule 1 and risks of future myocardial infarction in apparently healthy men. The Lancet, 1998.351 (9096): p. 88-92. 
37. de Lemos, J.A., C.H. Hennekens, and P.M. Ridker, Plasma concentration of soluble vascular cell adhesion molecule-1 and subsequent cardiovascular risk. Journal of the American College of Cardiology, 2000. 36 (2): p. $423-426$.

38. Ridker, P.M., J.E. Buring, and N. Rifai, Soluble P-selectin and the risk of future cardiovascular events. Circulation, 2001.103 (4): p. 491-495.

39. Malik, I., et al., Soluble adhesion molecules and prediction of coronary heart disease: a prospective study and meta-analysis. The Lancet, 2001. 358 (9286): p. 971-975.

40. Luc, G., et al., Circulating soluble adhesion molecules ICAM-1 and VCAM-1 and incident coronary heart disease: the PRIME Study.Atherosclerosis, 2003. 170 (1): p. 169-176.

41. Blankenberg, S., et al., Circulating cell adhesion molecules and death in patients with coronary artery disease. Circulation, 2001.104 (12): p. 1336-1342.

42. Khan, I., et al., Positive Association of Dietary Inflammatory Index with Incidence of Cardiovascular Disease: Findings from a Korean Population-Based Prospective Study. Nutrients, 2020. 12 (2): p. 588.

43. Pocovi-Gerardino, G., et al., Dietary Inflammatory Index Score and Cardiovascular Disease Risk Markers in Women with Systemic Lupus Erythematosus. Journal of the Academy of Nutrition and Dietetics, 2020.120 (2): p. $280-287$.

44. Vissers, L.E., et al., The relationship between the dietary inflammatory index and risk of total cardiovascular disease, ischemic heart disease and cerebrovascular disease: Findings from an Australian population-based prospective cohort study of women. Atherosclerosis, 2016. 253 : p. 164-170.

45. Boden, S., et al., Dietary inflammatory index and risk of first myocardial infarction; a prospective population-based study.Nutrition journal, 2017. 16 (1): p. 21.

46. Bondonno, N.P., et al., Dietary inflammatory index in relation to sub-clinical atherosclerosis and atherosclerotic vascular disease mortality in older women. British Journal of Nutrition, 2017.117 (11): p. $1577-1586$.

47. Tyrovolas, S., et al., Dietary inflammatory potential is linked to cardiovascular disease risk burden in the US adult population. International journal of cardiology, 2017. 240 : p. 409-413.

Table 1 characteristics of individuals by tertiles of dietary inflammatory potential (means+-SD)

\begin{tabular}{|c|c|c|c|c|c|}
\hline $\mathrm{P}$-value ${ }^{\mathrm{a}}$ & $\mathrm{P}$-value ${ }^{\mathrm{a}}$ & $\begin{array}{l}\text { Tertiles of } \\
\text { energy by DIP }\end{array}$ & $\begin{array}{l}\text { Tertiles of } \\
\text { energy by DIP }\end{array}$ & $\begin{array}{l}\text { Tertiles of } \\
\text { energy by DIP }\end{array}$ & Variables \\
\hline & & $\mathrm{T} 3=129$ & $\mathrm{~T} 2=134$ & $\mathrm{~T} 1=133$ & \\
\hline 0.04 & 0.04 & $34.44 \pm 7.27$ & $34.59 \pm 6.8$ & $36.47 \pm 7.4$ & Age(years) \\
\hline 0.49 & 0.49 & $63.2 \pm 10.32$ & $69.2 \pm 82.7$ & $63.0 \pm 8.7$ & Weight $(\mathrm{kg})$ \\
\hline 0.85 & 0.85 & $23.97 \pm 3.65$ & $24.0 \pm 3.80$ & $24.2 \pm 3.37$ & $\operatorname{BMI}\left(\mathrm{kg} / \mathrm{m}^{2}\right)^{\mathrm{b}}$ \\
\hline 0.89 & 0.89 & $81.04 \pm 10.82$ & $80.51 \pm 9.83$ & $81.02 \pm 9.91$ & $\mathrm{WC}(\mathrm{CM})$ \\
\hline 0.05 & 0.05 & $95 \pm 101$ & $78 \pm 77$ & $60 \pm 79$ & $\begin{array}{l}\text { PA(MET- } \\
\text { h/wk) }\end{array}$ \\
\hline 0.24 & 0.24 & $107 \pm 1.24$ & $109 \pm 1.20$ & $108 \pm 1.04$ & $\mathrm{SBP}^{1}(\mathrm{mmHg})$ \\
\hline 0.08 & 0.08 & $68 \pm 0.97$ & $70 \pm 1.05$ & $71 \pm 0.88$ & $\mathrm{DBP}(\mathrm{mmHg})$ \\
\hline 0.84 & 0.84 & 5.9 & 7.4 & 5.9 & $\begin{array}{l}\text { Current OCP } \\
\text { use }(\%)\end{array}$ \\
\hline 0.67 & 0.67 & 0.7 & 2.2 & 1.5 & $\begin{array}{l}\text { Current } \\
\text { corticosteroid } \\
\text { use }(\%)\end{array}$ \\
\hline 0.30 & 0.30 & 4.4 & 4.4 & 8.1 & $\begin{array}{l}\text { Menopausal } \\
(\%)\end{array}$ \\
\hline
\end{tabular}




\begin{tabular}{|c|c|c|c|c|c|}
\hline $\mathrm{P}$-value ${ }^{\mathrm{a}}$ & P-value ${ }^{a}$ & $\begin{array}{l}\text { Tertiles of } \\
\text { energy by DIP }\end{array}$ & $\begin{array}{l}\text { Tertiles of } \\
\text { energy by DIP }\end{array}$ & $\begin{array}{l}\text { Tertiles of } \\
\text { energy by DIP }\end{array}$ & Variables \\
\hline 0.87 & 0.87 & 73.9 & 73.5 & 71.3 & Married (\%) \\
\hline 0.55 & 0.55 & 43 & 36.9 & 37.7 & $\begin{array}{l}\text { Overweight or } \\
\text { obese }(\%)\end{array}$ \\
\hline 0.34 & 0.34 & 22.348 .928 .7 & 34.74025 .3 & 24.444 .431 .1 & $\begin{array}{l}\text { SES }(\%)^{c} \text { High } \\
\text { Medium Low }\end{array}$ \\
\hline $\begin{array}{l}\text { BMI: body } \\
\text { mass index, } \\
\text { WC: waist } \\
\text { circumference, } \\
\text { SBP: systolic } \\
\text { blood } \\
\text { pressure,DBP: } \\
\text { diastolic blood } \\
\text { pressure, } \\
\text { OCP: oral } \\
\text { contraceptives }\end{array}$ & $\begin{array}{l}\text { BMI: body } \\
\text { mass index, } \\
\text { WC: waist } \\
\text { circumference, } \\
\text { SBP: systolic } \\
\text { blood } \\
\text { pressure,DBP: } \\
\text { diastolic blood } \\
\text { pressure, } \\
\text { OCP: oral } \\
\text { contraceptives }\end{array}$ & $\begin{array}{l}\text { BMI: body } \\
\text { mass index, } \\
\text { WC: waist } \\
\text { circumference, } \\
\text { SBP: systolic } \\
\text { blood } \\
\text { pressure,DBP: } \\
\text { diastolic blood } \\
\text { pressure, } \\
\text { OCP: oral } \\
\text { contraceptives }\end{array}$ & $\begin{array}{l}\text { BMI: body } \\
\text { mass index, } \\
\text { WC: waist } \\
\text { circumference, } \\
\text { SBP: systolic } \\
\text { blood } \\
\text { pressure,DBP: } \\
\text { diastolic blood } \\
\text { pressure, } \\
\text { OCP: oral } \\
\text { contraceptives }\end{array}$ & $\begin{array}{l}\text { BMI: body } \\
\text { mass index, } \\
\text { WC: waist } \\
\text { circumference, } \\
\text { SBP: systolic } \\
\text { blood } \\
\text { pressure,DBP: } \\
\text { diastolic blood } \\
\text { pressure, } \\
\text { OCP: oral } \\
\text { contraceptives }\end{array}$ & $\begin{array}{l}\text { BMI: body } \\
\text { mass index, } \\
\text { WC: waist } \\
\text { circumference, } \\
\text { SBP: systolic } \\
\text { blood } \\
\text { pressure,DBP: } \\
\text { diastolic blood } \\
\text { pressure, } \\
\text { OCP: oral } \\
\text { contraceptives }\end{array}$ \\
\hline
\end{tabular}

1. achieved from chi-square for categorical variables and analysis of variance for continues variables.

2. High SES; socioeconomic status was defined based on income, educational level, family size, renting the house, being owner of the house or house area, being owner of the car and number of the cars and kind of the car(s), number of bedrooms and determination of who was in charge of the family.

3. Body mass index [?]25

Table 2. dietary inflammatory potential intake of participants after adjusted energy (mean $\pm \mathrm{SD})$

\begin{tabular}{llll}
\hline & Tertile of energy - energy adjusted DIP & Tertile of energy - energy adjusted DIP & Tertile of energy -energ \\
\hline Nutrients & $\mathbf{T 1}(\mathbf{n = 1 3 6 )}$ & $\mathbf{T 2}(\mathbf{n}=\mathbf{1 3 7})$ & $\mathbf{T 2}(\mathbf{n}=\mathbf{1 3 7})$ \\
Protein & $124 \pm 98$ & $135 \pm 139$ & $102 \pm 99$ \\
Fat & $103 \pm 17$ & $117 \pm 88$ & $102 \pm 21$ \\
Carbohydrate & $338 \pm 59$ & $321 \pm 61$ & $335 \pm 71$ \\
Thiamin & $3 \pm 2$ & $5 \pm 25$ & $2 \pm 2$ \\
Riboflavin & $1.8 \pm 0.51$ & $1.6 \pm 0.39$ & $1.4 \pm 0.47$ \\
Niacin & $18.9 \pm 3$ & $18.8 \pm 3$ & $18.4 \pm 5$ \\
Pyridoxine & $2.2 \pm 0.6$ & $2.6 \pm 8$ & $1.6 \pm 0.6$ \\
Folic acid & $410 \pm 121$ & $304 \pm 41$ & $226 \pm 54$ \\
Cobalamin & $4.7 \pm 2$ & $5 \pm 2$ & $4 \pm 2$ \\
Ascorbic acid & $281 \pm 111$ & $181 \pm 50$ & $126 \pm 40$ \\
Vitamin A & $1928 \pm 939$ & $1276 \pm 302$ & $887 \pm 335$ \\
Vitamin E & $64 \pm 16$ & $69 \pm 19$ & $65 \pm 21$ \\
Beta carotene & $1527 \pm 897$ & $904 \pm 291$ & $582 \pm 311$ \\
Vitamin D & $1.4 \pm 1.5$ & $2.2 \pm 11$ & $0.9 \pm 1.3$ \\
Selenium & $0.04 \pm 0.16$ & $0.48 \pm 5$ & $-0.01 \pm 0.17$ \\
Zinc & $10 \pm 4$ & $10 \pm 6$ & $8 \pm 4$ \\
Iron & $22 \pm 6$ & $25 \pm 49$ & $20 \pm 8$ \\
Tea & $356 \pm 263$ & $324 \pm 316$ & $242 \pm 220$ \\
Magnesium & $326 \pm 59$ & $267 \pm 35$ & $216 \pm 46$ \\
Onion & $54 \pm 34$ & $40 \pm 27$ & $29 \pm 19$ \\
Garlic & $1.9 \pm 0.17$ & $1.9 \pm 0.25$ & $2 \pm 0.002$ \\
Fiber & $10 \pm 3$ & $7 \pm 2$ & $5 \pm 1$ \\
& & &
\end{tabular}




\begin{tabular}{llll}
\hline & Tertile of energy - energy adjusted DIP & Tertile of energy - energy adjusted DIP & Tertile of energy -energ \\
\hline Caffeine & $82 \pm 57$ & $83 \pm 127$ & $59 \pm 50$ \\
Pepper & $10 \pm 7.3$ & $10 \pm 8.7$ & $8 \pm 8.5$ \\
SAFA & $25 \pm 8$ & $27 \pm 14$ & $23 \pm 9$ \\
PUFA & $40 \pm 9$ & $46 \pm 44$ & $39 \pm 11$ \\
MUFA & $32 \pm 8$ & $47 \pm 146$ & $30 \pm 9$ \\
CHOL & $234 \pm 85$ & $263 \pm 93$ & $232 \pm 99$ \\
\hline
\end{tabular}

SAFA; saturated fatty acid, PUFA; poly unsaturated fatty acid, MUFA; monounsaturated fatty acid. CHOL; cholesterol.

Table 3. Index of endothelial functions across tertile categories of dietary inflammatory potential

Tertile of energy- adjusted DIP

\begin{tabular}{lllll}
\hline & T1 $(\mathrm{n}=133)$ & T2(n=136) & T3(n=135) & P for trend \\
E-selectin $(\mathrm{ng} / \mathrm{L})$ & E-selectin $(\mathrm{ng} / \mathrm{L})$ & E-selectin $(\mathrm{ng} / \mathrm{L})$ & E-selectin $(\mathrm{ng} / \mathrm{L})$ & E-selectin $(\mathrm{ng} / \mathrm{L})$ \\
Crude & $81.6 \pm 4.5$ & $85.5 \pm 4.5$ & $93.7 \pm 4.5$ & 0.35 \\
Model I $^{\mathrm{b}}$ & $80 \pm 5.6$ & $88 \pm 5.3$ & $80 \pm 6.0$ & 0.57 \\
Model II $^{\mathrm{c}}$ & $79 \pm 5.6$ & $88 \pm 5.3$ & $81 \pm 6.1$ & 0.57 \\
Model III $^{\mathrm{d}}$ & $79 \pm 5.7$ & $88 \pm 5.8$ & $82 \pm 6.7$ & 0.67 \\
sICAM-1 $(\mathrm{mg} / \mathrm{L})$ & sICAM-1 $(\mathrm{mg} / \mathrm{L})$ & sICAM-1 $(\mathrm{mg} / \mathrm{L})$ & sICAM-1 $(\mathrm{mg} / \mathrm{L})$ & sICAM-1 $(\mathrm{mg} / \mathrm{L})$ \\
Crude & $221 \pm 6.68$ & $211 \pm 6.63$ & $213 \pm 6.65$ & 0.83 \\
Model I & $215 \pm 10.9$ & $225 \pm 10.2$ & $215 \pm 11.7$ & 0.98 \\
Model II & $215 \pm 10.9$ & $225 \pm 10.4$ & $215 \pm 10.8$ & 0.98 \\
Model III & $214 \pm 9.5$ & $213 \pm 9.6$ & $212 \pm 11.1$ & 0.92 \\
sVCAM-1 (mg/L) & sVCAM-1 (mg/L) & sVCAM-1 (mg/L) & sVCAM-1 (mg/L) & sVCAM-1 $(\mathrm{mg} / \mathrm{L})$ \\
Crude & $503 \pm 11.76$ & $482 \pm 11.63$ & $509 \pm 11.67$ & 0.49 \\
Model I & $479 \pm 23.2$ & $496 \pm 21.8$ & $515 \pm 25.3$ & 0.45 \\
Model II & $478 \pm 23.1$ & $490 \pm 22.0$ & $516 \pm 25.2$ & 0.45 \\
Model III & $477 \pm 24.3$ & $492 \pm 24.8$ & $502 \pm 28.9$ & 0.50 \\
\hline
\end{tabular}

1. Values are mean $\pm \mathrm{SE}$ in the tables and were compute by the use of ANCOVA.

2. Model I: adjusted for physical activity (MET-h/wk), marital status (categorical), socioeconomic status (categorical). energy intake, age, current corticoid steroids use (yes or no), menopausal status (yes or no), current OCP use (yes or no), systolic blood pressure, diastolic blood pressure.

3. Model II: Further adjusted for body mass index.

4. Model III: more adjusted for lipid profiles and blood sugar.

5. $p$-Value was calculated from linear regression of adhesion molecules (E-selectin, sICAM-1 and sVCAM1) on a categorical variable of dietary in index intake.

Figure 1. The E-selectin plasma concentration in tertiles (mean $\pm \mathrm{SEM}$ )

Figure 2. The ICAM-1 plasma concentration in tertiles (mean \pm SEM)

Figure 3. The sVCAM-1 plasma concentration in tertiles (mean \pm SEM) 
Figure 1

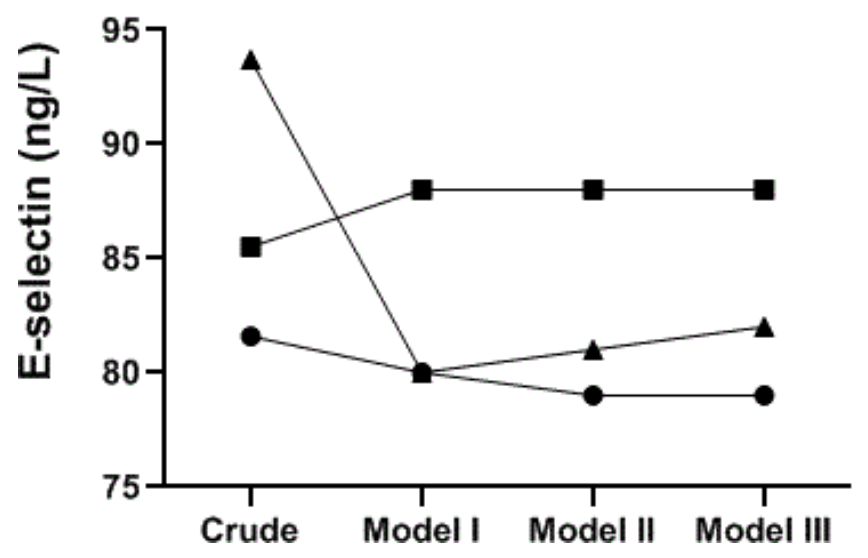

- Tertile $_{1}$
- Tertile $_{2}$
- Tertile 3

Figure 2

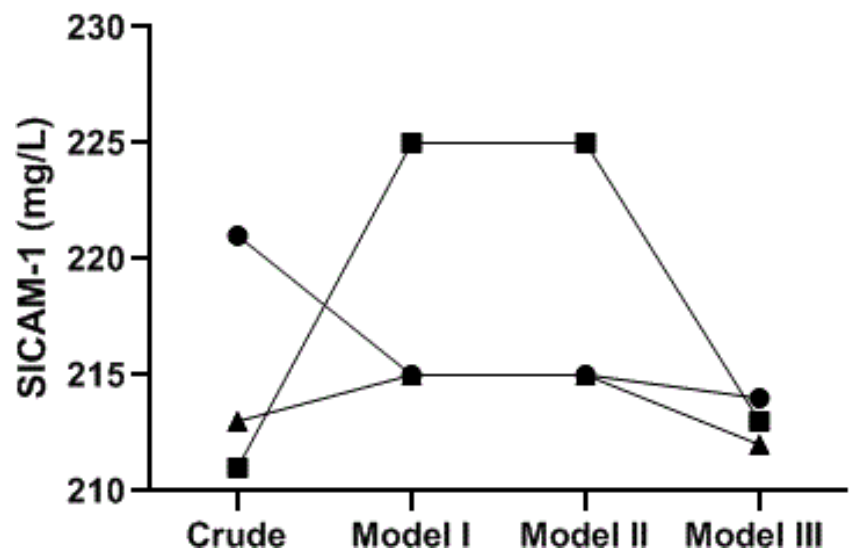

$$
\begin{aligned}
& - \text { Tertile }_{1} \\
& - \text { Tertile }_{2} \\
& \leftarrow \text { Tertile }_{3}
\end{aligned}
$$

Figure 3

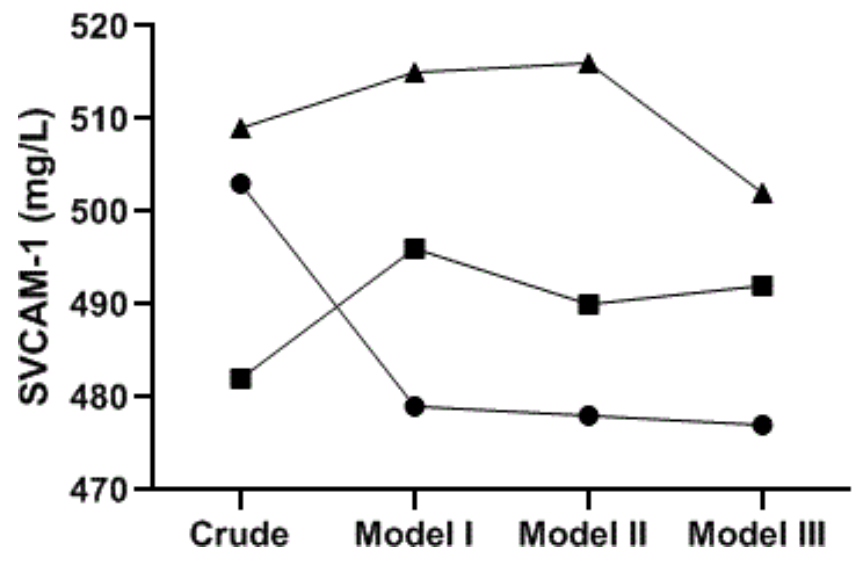

- Tertile $_{1}$

- Tertile $_{2}$

$\_$Tertile $_{3}$ 